\title{
HYDROGEN AND ITS COMPLEXES IN SILICON*
}

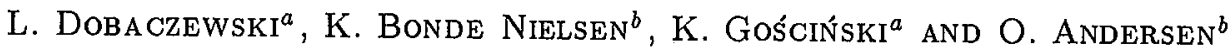 \\ ${ }^{a}$ Institute of Physics, Polish Academy of Sciences \\ Al. Lotników 32/46, 02-668 Warsaw, Poland \\ ${ }^{b}$ Institute of Physics and Astronomy, University of Aarhus, Aarhus, Denmark
}

In this study the technique of Laplace transform (high resolution) deep level transient spectroscopy combined with the uniaxial stress method has been used to study a symmetry and the defect reconfiguration kinetics (the stress induced alignment) of some forms of hydrogen-related centres. We have confirmed the trigonal symmetry of the defect related to the isolated bond centred hydrogen. When hydrogen decorates the vacancy-oxygen pair (the $A$ centre) the apparent defect orthorhombic symmetry is not lowered as a result of a very high hydrogen jumping rate between two unsaturated broken bonds of the vacancy. We also show that the stress-induced defect alignment in some cases can be related to the same microscopic mechanism of the hydrogen motion as it is for the diffusion process.

PACS numbers: $68.55 . \mathrm{Ln}, 71.55 . \mathrm{Cn}, 73.40 . \mathrm{Lq}$

Hydrogen in silicon may influence the material electrical characteristics through interactions with intrinsic defects, native impurities, and shallow donors or acceptors. This feature of hydrogen has important technological as well as fundamental aspects. For a longer time hydrogen has been studied extensively with a number of experimental and theoretical methods [1]. The structure of isolated hydrogen in crystalline silicon has been calculated by several groups [2, 3] and a general consensus has been reached that hydrogen in silicon can exist in three charge states: $\mathrm{H}^{+}, \mathrm{H}^{0}$, and $\mathrm{H}^{-}$. This impurity in the first two states is stable in the bond centred (BC) position, in the latter one in the interstitial tetrahedral site. Recently, it has been shown that two kinds of BC hydrogen donors exist and both of them can be only observed after proton implantation at low temperatures [4]. One is associated with the isolated hydrogen at the $\mathrm{BC}$ site, the other one with the BC hydrogen weakly perturbed by an interstitial oxygen. For temperatures above $240 \mathrm{~K}$ both forms of the defects disappear as the hydrogen atoms can migrate and create more stable complexes, e.g., with the vacancy-oxygen pair or carbon.

In this study the microscopic structure of different forms of hydrogen-related defects have been investigated with the use of the high resolution Laplace deep

*This work is supported in part by the Committee for Scientific Research (Poland) grant No. 8T11B00315 and by the Danish National Research Foundation through the Aarhus Centre for Atomic Physics (ACAP). 
level transient spectroscopy (DLTS) method [5] combined with the uniaxial stress. For the defects, or defect complexes, having the local symmetry lower than the $T_{d}$ symmetry the uniaxial stress affects the defect electronic level in a different way depending on the defect spatial orientation in respect to the stress. This effect results in a splitting of the defect electronic level. The DLTS technique investigates the process of the thermal emission of carriers bound by defects. The lifting of the defect spatial degeneracy can be only seen in this process when the defect reorientation rate is much slower than the thermal emission rate, otherwise the defect non-equivalent orientations are well averaged by the defect rotation and an average energy level is seen.

When the defect of a given symmetry is stressed along one of the major crystallographic directions $(\langle 100\rangle,\langle 110\rangle$ or $\langle 111\rangle)$ then the Laplace DLTS line can split and a number of split components can be related to the defect microscopic structure. For example, a defect with the $C_{3 v}$ local symmetry should reveal no splitting of the Laplace DLTS line when the sample is stressed in the $\langle 100\rangle$ direction, however this line should split into two lines with the amplitude ratios $1: 1$ or $3: 1$, when the stress is applied in the $\langle 110\rangle$ or $\langle 111\rangle$ direction, respectively. Although the number of split lines and the amplitude ratios are unique for the defect symmetry, the line separation and their stress coefficients should be related to details of the defect bond structure. In general, the Laplace DLTS technique allows one to observe much smaller modifications of the thermal emission process induced by the stress than it is possible for the conventional DLTS method. Usually, a difference in the emission rate by a factor of 2 or more is sufficient to see the spectral lines splitting.

When the thermal emission process occurs at the uniaxial stress it means that both, i.e. the initial and final, states of the process are modified by the stress. On the other hand, the thermal excitation of a carrier does not comply with any of selection rules, thus the final state of the process does not preserve any memory of the initial state symmetry. As a result, the symmetry of the final state is practically unknown and should be the same for all initial states. The final state of the process is seen in the experiment as stress-modified density of states of the conduction/valence band.

The samples used for one set of measurements have been cut from the same $1 \mathrm{~mm}$-thick wafer of Czochralski grown $n$-type silicon with the (110) surface orientation. This surface orientation allows one to prepare from the same crystal a set of stress-samples where the stress can be applied along three major crystallographic directions. For the isolated hydrogen studies the Schottky diodes were implanted with protons through the diode junction into the substrate at low temperature (around $60 \mathrm{~K}$ ). Test samples implanted with helium have been also prepared at equivalent (low) damage loads in order to distinguish between hydrogen-specific levels and intrinsic levels caused by the ion-beam damage. The implantation doses for the measurement and test samples were chosen to generate the same number of primary vacancies at the end of the range of the ions. For the studies of the hydrogen-related complexes the implantation was carried out at room temperature. These samples were compared with the reference samples irradiated with electrons. For all the samples the implantation energies were chosen so that the 
range matched the middle of the depletion layer at convenient for measurement reverse biases.

Figure 1 shows a series of the Laplace DLTS spectra taken at different uniaxial stresses applied along the $\langle 111\rangle$ direction to the $\mathrm{Si}(n) \mathrm{Cz}$ sample. This signal appeared after proton implantation and has been previously unambiguously identified as being due to the donor level of the $\mathrm{BC}$ isolated hydrogen (the $E 3^{\prime}$ level of $\mathrm{H}(\mathrm{BC}))$. The implantation procedure and the electronic level identification have been described in Ref. [4] in details. The subsequent spectra have been measured in $0.05 \mathrm{GPa}$ steps of stress and the vertical shift of a baseline is proportional to the stress. It is clearly seen that for this stress direction the Laplace DLTS peak splits into two components having the amplitude ratio equal to $3: 1$. In the $0.5 \mathrm{GPa}$ spectrum the most right hand side peak does not belong to the $\mathrm{H}(\mathrm{BC})$ centre but it is a split-partner of the broad line seen on the left hand side of the

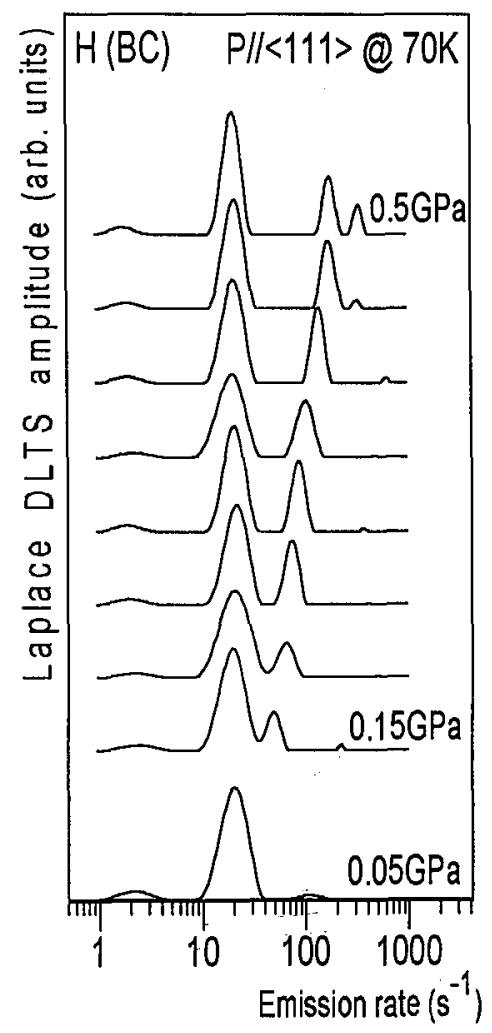

Fig. 1. The Laplace DLTS spectra taken at different uniaxial stresses applied along the (111) direction to the $\mathrm{Si}(n) \mathrm{Cz}$ sample. This signal appears after proton implantation and has been previously unambiguously identified as being due to the donor level of the bond-centred isolated hydrogen. The vertical shift of a baseline is proportional to the stress. 


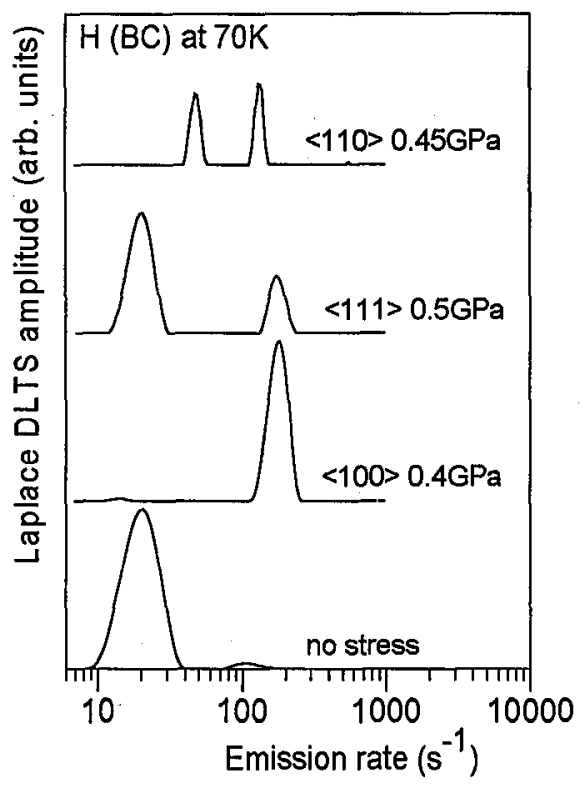

Fig. 2. The effect of the uniaxial stress on the Laplace DLTS spectrum of the isolated bond centred hydrogen (the $E 3^{\prime}$ donor state) for different stress directions.

$0.05 \mathrm{GPa}$ spectrum. This peak we associate to the presence of the $A$ centre (the vacancy-oxygen complex), which is observed in our samples with a much lower than $\mathrm{H}(\mathrm{BC})$ concentration. The $A$ centre line splits under the stress with much larger stress coefficients than $\mathrm{H}(\mathrm{BC})$ so its partner appears on the spectrum on the other side of the $\mathrm{H}(\mathrm{BC})$ peaks only at the highest stresses. For all stresses the sum of amplitudes of both lines attributed to the $\mathrm{BC}$ hydrogen equals to the amplitude of the unsplit line.

The similar stress experiments have been performed for the samples where the stress has been oriented along different crystallographic directions. Figure 2 shows a comparison of the spectra taken for different sample orientations. It is seen that, besides what has been already presented in Fig. 1, the Laplace DLTS peak does not split when the stress is applied in the $\langle 100\rangle$ direction and it splits into two components of equal amplitudes for the $\langle 110\rangle$ direction. The number of peaks and their amplitude ratios, observed for different sample orientations for the $E 3^{\prime}$ donor level, indicate the $C_{3 v}$ symmetry of the isolated bond centred hydrogen. In the consequence, the result is in agreement with the model of the defect. The symmetry of the bond centred hydrogen has been already postulated by Gorelkinskii and Nevinnyi basing upon the photo EPR measurements [6]. In our case the defect symmetry has been demonstrated for the donor energy level for the first time and correlated with the stability and diffusion studies performed in Ref. [4].

The microscopic structure of the $E 3^{\prime}$ level has been additionally confirmed by an analysis of the stress-induced defect alignment effect. In this experiment the stress is applied to the sample at higher than the measurement temperature 
(140-190 K) and the sample is then cooled down with the stress kept on. The diode bias is also kept on during the cooling process, which assures that the hydrogen is in the positive charge state. The temperatures where the stress is applied is chosen such that the hydrogen reorientation rate (a jump from one $\mathrm{BC}$ position to another) is sufficient for hydrogen to change the bond to occupy during a period of a few minutes of the annealing time. On the other hand, this temperature cannot be too high as hydrogen may diffuse as far as an average distance to a nearest trap, e.g. an oxygen atom.

It can be expected, and has been shown by the theory (see, e.g., Ref. [2]), that the hydrogen taking the $\mathrm{BC}$ position has to push aside both neighbouring silicon atoms. Consequently, if the bond accommodating hydrogen is externally stressed then one can expect that this would make this bond unfavourable for hydrogen to take when it jumps from one bond to another. This effect has been clearly seen when the alignment phenomenon is observed for the $\langle 111\rangle$ sample. In this case the 3:1 line splitting means that one bond (out of four possible) is parallel to the stress and remaining three are at a certain certain angle. The alignment process led here to a loss of the amplitude for the smaller line while the larger line used to gain the same amount of amplitude. For the $\langle 110\rangle$ sample, where the line splitting is 1:1 (actually $3: 3$ as there are six equivalent orientations of a defect with a trigonal symmetry and the stress), the defect configurations represented by the right hand side line in the spectrum seem to have a lower angle to the stress as this line has been loosing the amplitude during the alignment process, while the other three configurations (the left hand side line) have to have a larger angle to the stress as this line has been gaining the amplitude.

The isolated bond centred position of hydrogen is a highly unstable configuration. At temperatures above $110 \mathrm{~K}$ hydrogen in the neutral charge state starts to diffuse and can be easily trapped by the nearest oxygen. It seems that the perturbation of the bond centred hydrogen donor level $\left(E 3^{\prime \prime}\right)$ coming from oxygen is rather weak as both donor levels $\left(E 3^{\prime}\right.$ and $\left.E 3^{\prime \prime}\right)$ have exactly the same carrier emission characteristics. On the other hand, both of them differ very much in the stability and the diffusion properties (see Ref. [4] for details). The conversion of the oxygen perturbed bond centred hydrogen to the negative charge state results in the appearance of much deeper acceptor level. Such a sequence of the energy levels, i.e. the donor level above the acceptor level, is a consequence of the negative- $U$ character of the defect. This character of the isolated hydrogen in silicon has been postulated for a long time by the theory and practically never evidenced. Johnson et al. have observed an appearance of non-stable deep acceptor state immediately after the phosphorus-hydrogen pair breaking [7]. This experiment could not be reproduced independently [8]. Recently, it has been shown that the acceptor levels related to the isolated $\mathrm{BC}$ hydrogen and oxygen perturbed $\mathrm{BC}$ hydrogen should coexist as the donor levels attributed to both of them $\left(E 3^{\prime}\right.$ and $\left.E 3^{\prime \prime}\right)$ coexist as well [9].

When the Fermi level is above the $E 3^{\prime \prime}$ level the oxygen perturbed hydrogen immediately traps an extra electron and converts to a negatively charged defect. For hydrogen in the space charge of the diode one can easily observe this two-step capture process: $\mathrm{H}^{+}+2 e^{-} \rightarrow H^{0}+e^{-} \rightarrow H^{-}$and allows this process to occur 


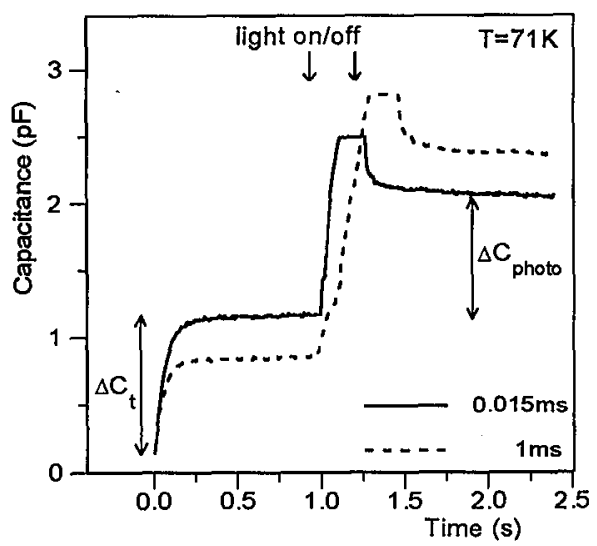

Fig. 3. The capacitance trace of the proton implanted $\mathrm{Si}(n) \mathrm{Cz}$ sample. The first transient of the $\Delta C_{t}$ amplitude starting at $t=0 \mathrm{~s}$ is related to the thermal emission from the $E 3^{\prime \prime}$ donor state, the steady-state capacitance step invoked by a short sample illumination (of the amplitude $\Delta C_{\text {photo }}$ ) is related to photoionisation of the hydrogen acceptor state. The solid and dashed lines were recorded after two different initial filling pulses: $0.015 \mathrm{~ms}$ and $1 \mathrm{~ms}$, respectively. See the text for details.

only during a certain time. The first step is usually very fast, the following is much slower and takes tenths of milliseconds. Varying the capture time it is thus possible to change a ratio of defects being in the neutral and negative charge state.

Figure 3 shows that when the oxygen perturbed $\mathrm{BC}$ hydrogen converts to a deeper energy state it indeed captures an extra electron. A number of defects being in the $E 3^{\prime \prime}$ state and in the deeper one is monitored by observation of amplitudes of the thermal and optical emission transients, respectively. In this experiment the temperature is chosen such that the thermal emission from the $E 3^{\prime \prime}$ donor state has an easily measurable value, but this temperature is much too low for the thermal emission from the deeper state to occur. The solid line shows a trace of the diode capacitance after $0.015 \mathrm{~ms}$ filing pulse. Immediately after the pulse, similarly to a conventional DLTS experiment, the thermal emission transient of the $\Delta C_{t}$ amplitude from $E 3^{\prime \prime}$ is seen. Next, a short white light illumination photoionises all defects remaining in the space charge region and deeper than $E 3^{\prime \prime}$. The amplitude of this photoionisation process is assigned in Fig. 3 as $\Delta C_{\text {photo. If any, but longer }}$ than the first one, filling pulse is applied (see the dashed line) then the amplitude of the first transient decreases, as the defect in the $E 3^{\prime \prime}$ state has more time to capture the second electron, and the amplitude of the photoionisation process increases. A simple comparison of the solid and dashed lines shows that an increase in $\Delta C_{\text {photo }}$ is twice bigger than the decrease in $\Delta C_{t}$. In separate experiments the energy of this deeper acceptor level of the oxygen perturbed hydrogen has been found to be equal to $0.68 \mathrm{eV}$ below the conduction band evidencing in this way the negative- $\mathrm{U}$ character of this defect [9].

All the above described experiments can be performed only if the sample is proton implanted at low temperature and the following measurements are realised in situ without taking the sample temperature much above the implantation 


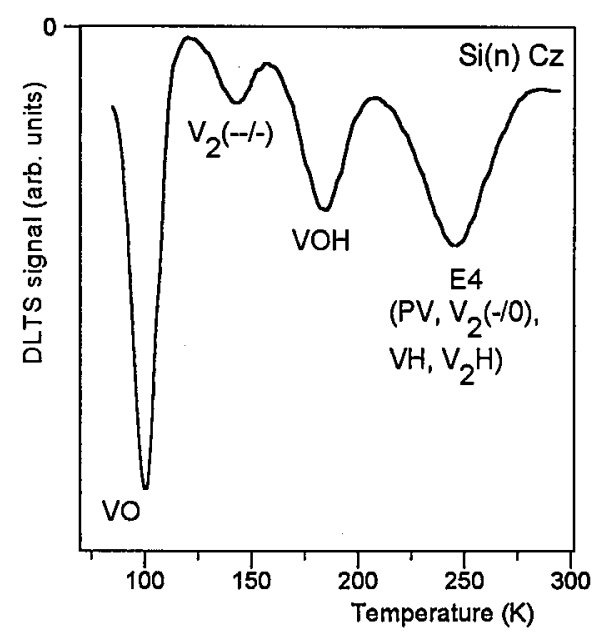

Fig. 4. Conventional DLTS spectrum of the $\mathrm{Si}(n) \mathrm{Cz}$ sample after a room temperature implantation. Different radiation defects, or groups of defects, are assigned.

temperature. When the implantation is done at room temperature then hydrogen is immediately trapped by vacancies or vacancy-related complexes formed in the same implantation process. Figure 4 shows the conventional DLTS spectrum of the proton implanted sample of the Czochralski grown $n$-type silicon. All of the peaks are associated to defects which are related to vacancies created in the crystal by the implantation process. The group of defects seen as the most right hand side peak has been already identified by us as different vacancy related complexes, some of them decorated with hydrogen [10]. Unfortunately, this group is too complex to perform unambiguous uniaxial stress experiments but its particular components can be identified by comparison with the annealing studies performed on the identified signals with EPR [11, 12].

The effect of the uniaxial stress on the thermal emission could be observed for the hydrogen decorated vacancy-oxygen (VO) complex (the $A$ centre). The structure of the $A$ centre was evidenced long time ago by Watkins and Corbett and its symmetry was found orthorhombic [13]. The DLTS signal related to the vacancy-oxygen-hydrogen ( $\mathrm{VOH}$ ) complex is observed at around $170 \mathrm{~K}$. At this temperature the reorientation rate of $\mathrm{VO}$ is much too high to give a chance to see the effect of the stress on the emission. However, it turned out that when VO binds an extra hydrogen atom the reorientation rate of the defect is retarded so much that the thermal emission of electrons from VOH becomes much more probable than the reorientation of the whole complex. As a result, the experimental conditions become favourable to observe the influence of stress on the emission.

Figure 5 shows how the Laplace DLTS peak splits under stress for different sample orientations. For the $\langle 111\rangle$ direction the peak splits into two equal lines, for the $\langle 110\rangle$ and $\langle 100\rangle$ directions the ratio of the peak amplitudes are 5:1 and $1: 2$, respectively. In all directions the amplitudes of the split lines sum up to the value of the amplitude observed for the unstressed sample. The observed pattern 


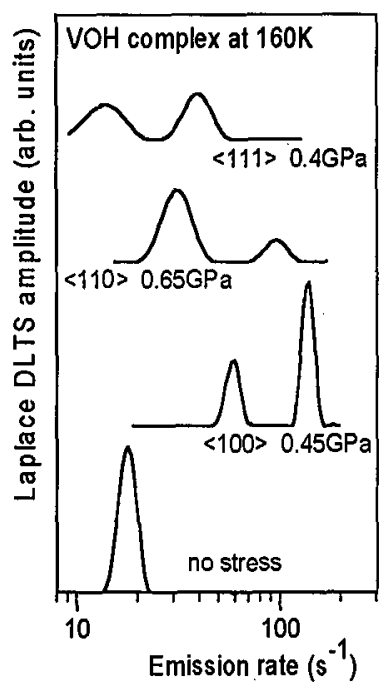

Fig. 5. The effect of the uniaxial stress on the Laplace DLTS spectrum of the VOH complex for different stress directions.

and intensity ratios of the stress-split components are characteristic for a centre with orthorhombic-I symmetry, i.e. similar to the VO complex. One could have expected that the extra atom in the complex should have lowered the overall defect symmetry, however, the recently identified EPR signal of the $A$ centre binding a single hydrogen atom displays the same orthorhombic symmetry [12] at this measurement temperature. The symmetry of the defect becomes indeed lower if the EPR signal is observed at much lower temperatures.

The vacancy in silicon leaves four broken bonds. In the VO complex two of them are saturated with an interstitial oxygen atom, and the remaining two bridge two silicon atoms along the $\langle 110\rangle$ direction. In the $\mathrm{VOH}$ complex one of the latter two bonds is saturated by hydrogen, the remaining one binds the electron. However, at temperatures around $160 \mathrm{~K}$ the hydrogen can very easily jump between these two silicon atoms along the $\langle 110\rangle$ direction and both, i.e. Laplace DLTS and EPR, experiments see its position averaged. This effect makes that the dynamic symmetries of the $\mathrm{VO}$ and $\mathrm{VOH}$ complexes observed at higher temperatures are the same.

In summary, hydrogen forms different types of defects in silicon depending on how it has been introduced into the crystal. The most simple form of them is the isolated hydrogen in the bond centred position. This form is only observed after proton implantation at low temperatures. The Laplace DLTS measurements under uniaxial stress confirm the trigonal symmetry of this defect. In the $n$-type silicon the BC hydrogen captures an extra electron forming a much deeper acceptor state stable up to at least $220 \mathrm{~K}$. This reversed sequence of the energy levels, i.e. the donor above the acceptor level, indicates the negative- $U$ character of the isolated and oxygen perturbed configurations of hydrogen. Finally, hydrogen can compensate various implantation created defects in silicon. In the intermedi- 
ate temperature range (well above the liquid nitrogen temperature) hydrogen, although bound by a defect, can reconfigure quickly within the unit cell showing the defect symmetry much higher than one would expect from a frozen configuration of atoms.

\section{References}

[1] For details see Hydrogen in Semiconductors, Eds. J.I. Pancove, N.M. Johnson, in series Semiconductors and Semimetals, Vol. 34, Academic, Boston 1991; S.J. Pearton J.W. Corbett, M. Stavola, Hydrogen in Crystalline Semiconductors, Springer-Verlag, Berlin 1992 and references therein.

[2] S.K. Estreicher, Phys. Rev. B 36, 9122 (1987).

[3] C.G. Van de Walle, P.J.H. Denteneer, Y. Bar Yam, S.T. Pantelides, Phys. Rev. B 39, 10791 (1989); R. Jones, Physica B 170, 181 (1991); K.J. Chang, D.J. Chadi, Phys. Rev. Lett. 62, 937 (1989).

[4] K. Bonde Nielsen, B. Bech Nielsen, J. Hansen, E. Andersen, J.U. Andersen, Phys. Rev. B 60, 1716 (1999).

[5] L. Dobaczewski, P. Kaczor, I.D. Hawkins, A.R. Peaker, J. Appl. Phys. 76, 194 (1994).

[6] Yu.V. Gorelkinskii, N.N. Nevinnyi, Pisma Zh. Tekh. Fiz. 13, 105 (1987) [Sov. Tech. Phys. Lett. 13, 45 (1987)]; Physica B 170, 155 (1991).

[7] N.M. Johnson, C. Herring, C.G. Van de Walle, Phys. Rev. Lett. 73, 130 (1994).

[8] C.H. Seager, R.A. Anderson, S.K. Estreicher, Phys. Rev. Lett. 74, 4562 (1995).

[9] K. Bonde Nielsen, L. Dobaczewski, S. Søgård, B. Bech Nielsen, unpublished.

[10] K. Bonde Nielsen, L. Dobaczewski, K. Gościnski, R. Bendesen, O. Andersen, B. Bech Nielsen, Physica B 273, 167 (1999).

[11] B. Bech Nielsen, P. Johannesen, P. Stallinga, K. Bonde Nielsen, J.R. Byberg, Phys. Rev. Lett. 79, 1507 (1997); P. Stallinga, P. Johannesen, S. Herström, K. Bonde Nielsen, B. Bech Nielsen, J.R. Byberg, Phys. Rev. B 58, 3842 (1998).

[12] P. Johannesen, B. Bech Nielsen, J.R. Byberg, Physica B 273, 180 (1999).

[13] G.D. Watkins, J.W. Corbett, Phys. Rev. 121, 1001 (1961). 